\title{
Hábito alimentar do peixe-espada adulto, Trichiurus lepturus, na costa norte do Rio de Janeiro, sudeste do Brasil
}

\author{
Vanessa Trindade Bittar* \\ Bernardo de Figueiredo Leão Castello \\ Ana Paula Madeira Di Beneditto \\ Universidade Estadual do Norte Fluminense/UENF, Laboratório de Ciências Ambientais/LCA \\ Centro de Biociências e Biotecnologia/CBB, Av. Alberto Lamego, 2.000, CEP 28013-602 \\ Campos dos Goytacazes - RJ, Brasil \\ *Autora para correspondência \\ vatrindade@gmail.com
}

Submetido em 18/09/2007

Aceito para publicação em 10/01/2008

\section{Resumo}

O presente estudo tem como objetivo caracterizar o hábito alimentar do peixe-espada adulto, Trichiurus lepturus, no norte do Rio de Janeiro através da avaliação de conteúdos estomacais. A amostragem incluiu 350 espécimes, com comprimento total entre 100 e $163 \mathrm{~cm}$, coletados entre 2004 e 2006 . Para cada espécie de presa calcularam-se as freqüências de ocorrência, numérica e de biomassa, porte (comprimento e peso) e Índice de Importância Relativa (IIR) na dieta. O comportamento alimentar de canibalismo foi registrado. De acordo com o IIR, os itens preferenciais foram os peixes T. lepturus, Pellona harroweri, Chirocentrodon bleekerianus, Lycengraulis grossidens, Peprilus paru, Chloroscombrus chrysurus, Odontognathus mucronatus, Stellifer brasiliensis e Isophisthus parvipinnis, além do crustáceo Pleoticus muelleri. Os cefalópodes Loligo plei e L. sanpaulensis ocorreram ocasionalmente nos conteúdos estomacais analisados. Na costa norte do Rio de Janeiro, T. lepturus apresenta dieta composta por organismos neríticos de hábitos pelágicos e/ou demersais associados a áreas costeiras e estuarinas, sendo peixes e crustáceos os itens mais representativos na dieta.

Unitermos: Trichiurus lepturus, peixe-espada, hábito alimentar, canibalismo, norte do Rio de Janeiro

\section{Abstract}

Feeding habits of the cutlassfish, Trichiurus lepturus, in northern Rio de Janeiro. The aim of the present study was to describe the diet of the adult cutlassfish, Trichiurus lepturus, in northern Rio de Janeiro, through stomach content analysis. The sampling included 350 specimens measuring between 100 and $163 \mathrm{~cm}$ in total length, collected from 2004 to 2006 . For each prey species, the frequency of occurrence, density and biomass, body length and weight, and Index of Relative Importance (IRI) were calculated. The cannibalistic feeding behavior of the cutlassfish was also recorded. According to the IRI, the preferential prey species were the fishes T. lepturus, Pellona harroweri, Chirocentrodon bleekerianus, Lycengraulis grossidens, Peprilus paru, Chloroscombrus chrysurus, Odontognathus mucronatus, Stellifer brasiliensis and Isophisthus parvipinnis, and the crustacean Pleoticus muelleri. The cephalopods Loligo sanpaulensis and L. plei occurred occasionally in the stomach contents analyzed. The diet of T. lepturus in northern Rio de Janeiro was composed of pelagic and demersal prey species, associated with estuarine and coastal areas. The fish species and one crustacean species were the most representative items in its diet.

Key words: Trichiurus lepturus, cutlassfish, feeding habits, cannibalism, northern Rio de Janeiro 


\section{Introdução}

Trichiurus lepturus, L. 1758 (Perciformes, Trichiuriidae) é uma espécie cosmopolita denominada comumente de peixe-espada ou espada. Distribui-se em águas quentes e temperadas, entre $60^{\circ} \mathrm{Ne} 45^{\circ} \mathrm{S}$, com salinidades entre 33 e $36 \mathrm{ppm}$ e temperaturas superiores à $16^{\circ} \mathrm{C}$. No Oceano Atlântico, a espécie se distribui do Canadá $\left(\sim 40^{\circ} \mathrm{N}\right)$, até a Argentina $\left(\sim 37^{\circ} \mathrm{S}\right)$, desde a linha de costa até profundidades em torno de 350m (Magro et al., 2000; Martins e Haimovici, 2000; FAO, 2005; Magro, 2006). Apresenta hábito demerso-pelágico e é considerado oportunista e voraz quanto à alimentação (Martins e Haimovici, 2000; FAO, 2005; Chiou et al., 2006).

A espécie é predominantemente piscívora, inclusive pratica o canibalismo, mas cefalópodes e crustáceos peneídeos também fazem parte de sua dieta (Wojciechowski, 1972; Martins et al., 2005; Chiou et al., 2006). Apresenta heterogeneidade espacial e temporal influenciadas pela disponibilidade de alimento (Martins et al., 2005). No litoral do Rio Grande do Sul, sul do Brasil, fêmeas adultas e os juvenis permanecem na região da plataforma continental durante o inverno para se alimentarem e os machos adultos movimentam-se além dos limites da plataforma (Martins e Haimovici, 2000). A proximidade da costa acentua a intensidade alimentar para suprir as necessidades energéticas associadas ao crescimento, a reprodução e a desova (Martins e Haimovici, 2000; Chiou et al., 2006; Magro, 2006).

Trichiurus lepturus está entre as seis espécies com maior volume de desembarque pesqueiro mundial (Martins e Haimovici, 1997; FAO, 2005). Além da importância comercial em algumas regiões, apresenta relevância ecológica. A posição trófica dos indivíduos adultos dessa espécie é imediatamente subseqüente àquela ocupada por elasmobrânquios e pequenos cetáceos (Chiou et al. 2006). No litoral sudeste e sul do Brasil, o peixe-espada já foi registrado como item alimentar desses animais (Di Beneditto et al., 2001; Santos et al., 2002; Aguiar, 2003), mas também se levanta a possibilidade de que seja um potencial competidor trófico do pequeno cetáceo Pontoporia blainvillei, que se distribui em regiões costeiras e vem sofrendo declínio populacional devido a ação antrópica (Bassoi, 1997 e 2005; Di Beneditto et al., 2001).
A ausência de determinadas espécies de peixes na pesca comercial praticada em determinadas regiões do litoral brasileiro pode ser decorrente da exclusão trófica causada devido a presença de T. lepturus (Magro et al., 2000). No sul do Brasil, grandes concentrações de T. lepturus estão associadas ao declínio de espécies de peixes comercialmente importantes, tais como Micropogonias furnieri (corvina), Umbrina canosai (castanha) e Cynoscion guatucupa (pescada) (Martins e Haimovici, 1997).

Apesar da possibilidade de declínio populacional de algumas espécies de peixes na presença de $T$. lepturus e da possível competição trófica com Pontoporia blainvillei, não há informações referentes a sua dieta, bem como aos seus hábitos alimentares e suas inter-relações no litoral norte do Rio de Janeiro. Dessa forma, o presente estudo investiga pela primeira vez o hábito alimentar de T. lepturus na região, com o intuito de contribuir para o entendimento da dinâmica trófica envolvendo esse peixe e auxiliar futuros estudos sobre a ecologia da espécie e sua estruturação trófica, bem como de suas presas e potenciais competidores tróficos.

\section{Material e Métodos}

Os limites geográficos considerados para a costa norte do Rio de Janeiro são a localidade de Barra do Itabapoana ( $\left.21^{\circ} 18^{\prime} \mathrm{S}\right)$, no município de São Francisco do Itabapoana, e o município de Macaé $\left(22^{\circ} 25^{\prime}\right.$ 'S) (Muehe e Valentini, 1998) (Figura 1). As coletas dos espécimes estudados foram realizadas a partir da dinâmica usual das pescarias praticadas com redes de espera por embarcações sediadas no porto de Atafona (213ㄱ'S) (Figura 1). De modo geral, o campo de pesca dessas embarcações está compreendido entre os limites da costa norte do estado do Rio de Janeiro e as redes de espera são lançadas de menos de uma (1) a cerca de 42 milhas náuticas da linha de costa, em profundidades que variam entre $5 \mathrm{e}$ 70m (Di Beneditto, 2003).

Os espécimes adultos de T. lepturus medindo entre 100 e $163 \mathrm{~cm}$ de comprimento total foram coletados mensalmente entre agosto de 2004 e julho de 2006. No total, avaliou-se o conteúdo estomacal de 350 indivíduos como amostra representativa do hábito alimentar da espécie na região. 
A necropsia dos peixes foi realizada ainda nos entrepostos de pesca. O comprimento total em $\mathrm{cm}$ foi inferido com o auxílio de fita métrica e em seguida os animais foram abertos na porção ventral com o auxílio de tesoura e pinça. Posteriormente, os estômagos foram retirados da cavidade abdominal através de cortes em sua porção inicial e terminal e cada estômago foi acondicionado em saco plástico contendo solução de álcool aquoso $70 \%$.

Em laboratório, o conteúdo estomacal recuperado foi triado, identificado e quantificado sob estereomicroscópio com auxílio de coleções de referência das espécies de presas potenciais que se distribuem na região (Di Beneditto et al., 2001) e guias taxonômicos específicos (Boschi, 1963; Figueiredo e Menezes, 1978; 1980 e 2000; Menezes e Figueiredo, 1980 e 1985; Clarke, 1986a e 1986b; Cérvigon et al., 1993; Costa et al., 2003).

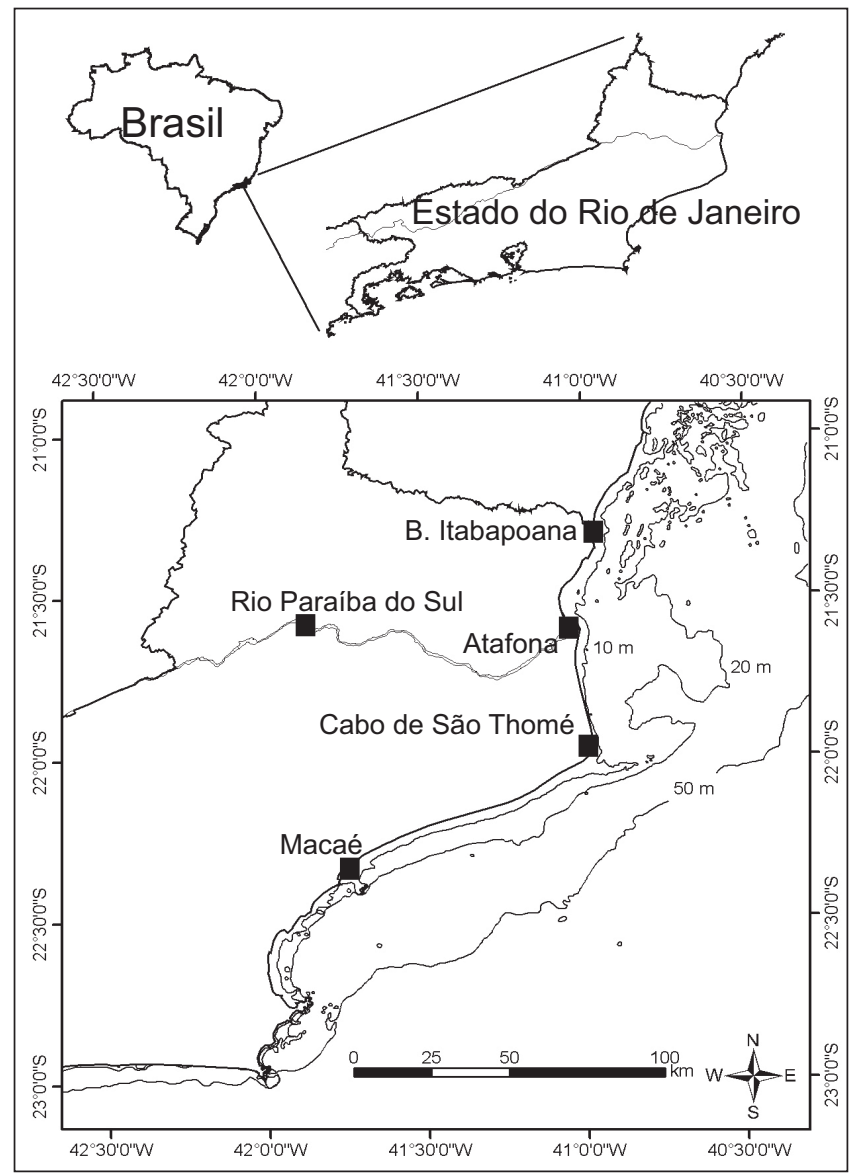

FIGURA 1: Mapa do Rio de Janeiro, destacando a costa norte e seus limites geográficos (Barra de Itabapoana e Macaé), o rio Paraíba do Sul, o porto de Atafona, o Cabo de São Thomé e o perfil batimétrico da região costeira.
A análise das presas (peixes, cefalópodes e crustáceos) recuperadas nos estômagos levou em consideração a quantidade predada (densidade $=\mathrm{n}^{\mathrm{o}}$ de indivíduos e biomassa consumida em gramas) e o seu porte (comprimento do corpo em $\mathrm{cm}$ e biomassa individual em gramas). A biometria dos itens recuperados, tais como otólitos de peixes, bicos de cefalópodes e cefalotórax de crustáceos, foi realizada sob estereomicroscópio com ocular micrométrica acoplada. As relações biométricas entre as estruturas supracitadas e o porte dos organismos permitiram estimativas quanto às dimensões das presas consumidas a partir de equações de regressão (Bastos, 1990; Di Beneditto et al., 2001; Bassoi, 2005).

Para cada espécie de presa calculou-se o percentual de freqüência numérica $(\% \mathrm{FN})$, definido como a densidade da presa (número de indivíduos) dividida pela densidade total de presas consumidas, o percentual de biomassa $(\% \mathrm{~W})$, definido como a biomassa da presa dividida pela biomassa total de presas ingeridas e o percentual de freqüência de ocorrência (\% FO), definido como o número de estômagos em que a presa ocorre pelo número total de estômagos com presença de itens alimentares. A importância de cada espécie de presa na dieta foi determinada através do Índice de Importância Relativa (IIR) (Pinkas et al., 1971): IIR $=[(\% \mathrm{FN}+\% \mathrm{~W})$ $\mathrm{x} \% \mathrm{FO}]$.

Devido às diferenças das presas quanto a digestibilidade e preservação de estruturas com relevância taxonômica e biométrica no conteúdo estomacal do predador, conforme relatado por Fitch e Brownell (1968) e Clarke (1986b), optou-se por calcular o IIR separadamente para cada grupo de presas (peixes, cefalópodes e crustáceos). Dessa forma, subestimativa ou superestimativa quanto à importância das espécies de presas puderam ser minimizadas. O presente estudo considera que as estimativas de porte, biomassa e densidade ( $\mathrm{n}^{\mathrm{o}}$ de indivíduos) das presas representam as quantidades mínimas consumidas pelo T. lepturus.

\section{Resultados}

A caracterização da alimentação de T. lepturus adultos no norte do Rio de Janeiro baseou-se na análise de 350 conteúdos estomacais de espécimes com compri- 
mentos totais entre 100 e $163 \mathrm{~cm}$ (média $=126 \pm 12,36$ ). Deste total, 343 conteúdos continham itens alimentares e puderam ser utilizados na análise da dieta. Vinte e oito espécies de presas perfazendo 1.676 indivíduos e $5.710 \mathrm{~g}$ foram registradas a partir dos estômagos com presença de itens alimentares.

O percentual da densidade, biomassa (g) e freqüência de ocorrência relativa dos grupos de espécies de presas na dieta de $T$. lepturus está indicado na figura 2. A maior densidade foi registrada para o grupo dos crustáceos, e os peixes corresponderam ao maior percentual da biomassa e da freqüência de ocorrência nos estômagos.

Os peixes registrados em torno de $58 \%(\mathrm{n}=200)$ dos estômagos (Figura 2) incluíram 23 espécies (Tabela 1). Cerca de $65 \%$ das espécies de peixes identificadas como presas são representantes das famílias Sciaenidae, Engraulidae e Clupeidae. As espécies T. lepturus, Pellona harroweri, Chirocentrodon bleekerianus, Lycengraulis grossidens, Peprilus paru, Chloroscombrus chrysurus, Odontognathus mucronatus, Stellifer brasiliensis e Isophisthus parvipinnis totalizaram $96,2 \%$ do somatório do IIR e destacaram-se, nessa ordem, como os principais peixes consumidos (Tabela 1).

Do total de peixes, doze apresentam hábito demersal, oito são pelágicos e três podem ser considerados como demerso-pelágicos. Com exceção de Pagrus pagrus, os peixes consumidos são caracteristicamente neríticos e $65 \%$ do total estão associados a regiões estuarinas. Dentre as quatro principais espécies, $P$. harroweri, $C$. bleekerianus e L. grossidens possuem hábitos pelágicos, sendo as duas últimas associadas a estuários (Figueiredo e Menezes, 1978; 1980 e 2000; Menezes e Figueiredo, 1980 e 1985; Di Beneditto, 2000), enquanto T. lepturus apresenta hábitos demerso-pelágicos (FAO, 2005).

O comportamento de canibalismo foi registrado e revelou o consumo de co-específicos com até $100 \mathrm{~cm}$ de comprimento. Entretanto, a média do comprimento da maior parte dos peixes consumidos não ultrapassou cerca de $10 \mathrm{~cm}$ (Tabela 1). A maior amplitude de densidade de uma determinada espécie de peixe por estômago foi verificada para $P$. harroweri (1-5 indivíduos) e de biomassa para T. lepturus $(0,2-649 \mathrm{~g})$.

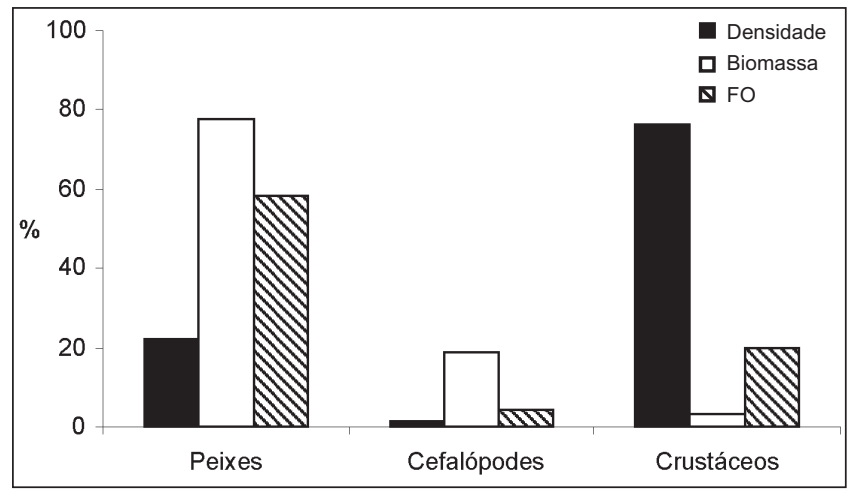

FIGURA 2: Percentual da densidade ( $\mathrm{n}^{\circ}$ de indivíduos), da biomassa (g) e da freqüência de ocorrência relativa dos grupos de presas registradas no conteúdo estomacal de Trichiurus lepturus na costa norte do Rio de Janeiro, entre agosto de 2004 e julho de 2006 ( $n=343$ conteúdos estomacais).

Os crustáceos foram registrados em 19,8\% ( $\mathrm{n}=$ 68) dos estômagos (Figura 2). Pleoticus muelleri, Xiphopenaeus kroyeri e Artemesia longinaris estavam presentes nos conteúdos estomacais, e a primeira espécie foi a mais representativa (Tabela 1), sendo as referidas espécies consideradas bentônicas (Di Beneditto, 2000; Costa et al., 2003). A média do comprimento da maioria das presas ficou em torno de $3 \mathrm{~cm}$ e a maior amplitude de densidade e de biomassa por estômago foi registrada para P. muelleri (1-178 indivíduos e 0,1$37 \mathrm{~g}$, respectivamente).

Os cefalópodes podem ser considerados como itens secundários na alimentação, pois foram registrados em apenas 4,3\% $(\mathrm{n}=15)$ dos estômagos (Figura 2). Foram identificadas as espécies Loligo plei e $L$. sanpaulensis, com o maior consumo da primeira, correspondendo a $96 \%$ do IIR referente aos cefalópodes (Tabela 1). As referidas espécies apresentam hábitos demerso-pelágicos (Roper et al., 1984). O comprimento do manto da maioria dos indivíduos não ultrapassou $10 \mathrm{~cm}$ e a maior amplitude de densidade e biomassa por estômago foi observada para L. plei (1-3 indivíduos e 2-193g, respectivamente). 
TABELA 1: Percentual de freqüência numérica $(\% \mathrm{FN})$, percentual de biomassa $(\% \mathrm{~W})$, percentual de freqüência de ocorrência (\%FO), Índice de Importância Relativa (IIR) e o comprimento total (CT) das espécies de presas consumidas por Trichiurus lepturus na costa norte do Rio de Janeiro entre agosto de 2004 e julho de $2006(n=343)$.

\begin{tabular}{|c|c|c|c|c|c|c|c|c|}
\hline \multirow{2}{*}{ PRESAS } & \multirow{2}{*}{$\% \mathrm{FN}$} & \multirow{2}{*}{$\% \mathrm{~W}$} & \multirow{2}{*}{$\%$ FO } & \multirow{2}{*}{ IIR } & \multicolumn{4}{|c|}{ CT (cm) } \\
\hline & & & & & Min & Máx & Média & dp \\
\hline \multicolumn{9}{|l|}{ Peixes } \\
\hline Trichiurus lepturus & 4,4 & 40,2 & 8,0 & 356,5 & 3,4 & 100,0 & 43,7 & 38,0 \\
\hline Pellona harroweri & 12,3 & 8,5 & 14,0 & 290,6 & 4,5 & 11,4 & 7,8 & 1,7 \\
\hline Chirocentrodon bleekerianus & 6,5 & 9,8 & 9,0 & 146,9 & 6,4 & 16,5 & 10,6 & 3,4 \\
\hline Lycengraulis grossidens & 3,3 & 9,0 & 6,0 & 73,4 & 5,2 & 24,6 & 9,5 & 5,8 \\
\hline Peprilus paru & 6,0 & 2,4 & 8,0 & 67,0 & 1,3 & 5,3 & 4,7 & 2,2 \\
\hline Chloroscombrus chrysurus & 2,2 & 4,2 & 4,0 & 25,6 & 28,9 & 32,9 & 31,0 & 1,5 \\
\hline Odontognathus mucronatus & 2,7 & 1,8 & 4,5 & 20,5 & 5,4 & 17,3 & 9,8 & 4,6 \\
\hline Stellifer brasiliensis & 2,7 & 1,6 & 3,0 & 12,8 & 2,6 & 7,6 & 4,6 & 1,9 \\
\hline Isophisthus parvipinnis & 1,4 & 2,4 & 2,5 & 9,5 & 6,0 & 14,6 & 10,4 & 3,5 \\
\hline Paralonchurus brasiliensis & 0,8 & 6,1 & 1,0 & 6,9 & 4,7 & 22,6 & 13,6 & 12,7 \\
\hline Bagre bagre & 1,1 & 3,5 & 1,5 & 6,8 & 1,1 & 17,4 & 9,3 & 11,5 \\
\hline Anchoa filifera & 1,6 & 0,5 & 2,5 & 5,4 & 3,9 & 6,0 & 4,7 & 0,9 \\
\hline Cynoscion jamaicensis & 0,5 & 3,9 & 1,0 & 4,5 & 7,5 & 13,9 & 10,7 & 4,6 \\
\hline Anchoviela lepidentostole & 1,4 & 0,3 & 2,5 & 4,1 & 5,3 & 6,6 & 5,8 & 0,6 \\
\hline Arius spixii & 1,9 & 0,1 & 2,0 & 4,0 & 0,8 & 5,2 & 3,0 & 3,2 \\
\hline Orthopristis ruber & 0,5 & 1,7 & 1,0 & 2,2 & 12,4 & 12,4 & 12,4 & 12,4 \\
\hline Stellifer sp. & 1,6 & 0,3 & 1,0 & 1,9 & 1,7 & 5,7 & 4,1 & 2,1 \\
\hline Pagrus pagrus & 0,3 & 2,5 & 0,5 & 1,4 & 8,9 & 8,9 & 8,9 & 8,9 \\
\hline Trachurus lathami & 0,5 & 0,4 & 1,0 & 0,9 & 7,1 & 17,2 & 12,2 & 7,1 \\
\hline Prionotus punctatus & 0,5 & 0,1 & 1,0 & 0,6 & 0,3 & 4,9 & 2,8 & 2,3 \\
\hline Porichthys porosissimus & 0,3 & 0,9 & 0,5 & 0,6 & 15,6 & 15,6 & 15,6 & 15,6 \\
\hline Anchoa sp. & 0,3 & 0,0 & 0,5 & 0,1 & 6,0 & 6,0 & 6,0 & 6,0 \\
\hline Macrodon ancylodon & 0,3 & 0,0 & 0,5 & 0,1 & $*$ & $*$ & $*$ & * \\
\hline \multicolumn{9}{|l|}{ Crustáceos } \\
\hline Pleoticus muelleri & 96,3 & 95,5 & 42,9 & 8222,9 & 2,7 & 3,5 & 3,1 & 0,4 \\
\hline Artemesia longinaris & 3,1 & 0,5 & 8,6 & 30,6 & 4,3 & 4,3 & 4,3 & 4,3 \\
\hline Xiphopenaeus kroyeri & 0,3 & 0,6 & 8,6 & 7,3 & 3,2 & 5,5 & 4,4 & 1,6 \\
\hline \multicolumn{9}{|l|}{ Cefalópodes } \\
\hline Loligo plei & 59,1 & 99,0 & 83,3 & 13173,7 & 1,0 & 24,0 & 10,2 & 7,6 \\
\hline Loligo sanpaulensis & 22,7 & 1,6 & 25,0 & 608,7 & 1,75 & 11,5 & 6,5 & 4,5 \\
\hline
\end{tabular}

$\mathrm{IIR}=[(\% \mathrm{FN}+\% \mathrm{~W}) \times \mathrm{FO}] ; \mathrm{dp} ;$ desvio padrão; * porte não estimado. 


\section{Discussão}

No norte do Rio de Janeiro $\left(\sim 22^{\circ} \mathrm{S}\right)$, T. lepturus adultos exploraram preferencialmente as águas costeiras para obtenção dos recursos alimentares. Seu hábito alimentar foi predominantemente piscívoro, apesar do registro de crustáceos e cefalópodes, predando preferencialmente presas pelágicas, fato também registrado por Wojciechowski (1972) na Mauritânia, Martins et al. (2005) e Magro (2006) no Brasil, além de Chiou et al. (2006) em Taiwan. A piscivoria associada principalmente aos organismos pelágicos pode ser uma estratégia para minimizar o tempo de forrageamento, favorecendo o ganho energético e/ou nutricional, e/ou ser devido a maior facilidade de captura desses animais durante seu deslocamento. Além disso, as presas mais abundantes na região (Di Beneditto, 2000) compõem a dieta de T. lepturus, o que demonstra a sua plasticidade alimentar. Essa amplitude da dieta pode estar associada a adaptação da espécie as mais variadas condições ambientais e já foi registrada no sul do Brasil (Martins et al., 2005) e em Taiwan (Chiou et al., 2006).

Os peixes das famílias Scianidae, Engraulidae e Clupeidae foram os mais representativos, com destaque para seus co-específicos, além de $P$. harroweri, $C$. bleekerianus e L. grossidens. No entanto, ao comparar as espécies predadas na região com aquelas encontradas no sul do Brasil, nota-se uma composição específica distinta entre essas áreas. No sul do Brasil $\left(\sim 32^{\circ} \mathrm{S}\right)$, Engraulis anchoita, T. lepturus e C. guatucupa são as presas preferenciais (Martins et al., 2005). Variações na composição específica da dieta podem ser reflexos da composição faunística distinta entre as áreas geográficas que compõem a distribuição da espécie. Essa preferência intraespecífica pode estar refletindo não apenas essa disponibilidade, mas também a habilidade comportamental em localizar, selecionar, capturar, ingerir e digerir esses organismos e/ou ser resultante da competição intraespecífica e/ou interespecífica pelo alimento ao longo da distribuição da espécie.

Trichiurus lepturus selecionou peixes com porte em torno de 7 a $10 \mathrm{~cm}$, dimensões de presas também registradas em outras regiões (ver em Martins et al., 2005). A seleção do porte pode estar associada ao cres- cimento somático, reprodução, desova e a maior habilidade de locomoção e desenvolvimento morfológico dos adultos de T. lepturus. Martins et al. (2005) relatam que a maior fonte de energia para essa espécie no sul do Brasil são presas pelágicas de pequeno porte, embora presas grandes também façam parte da sua alimentação, sendo este padrão o mesmo registrado para a região estudada.

No norte do Rio de Janeiro verificou-se o comportamento de canibalismo, fato já reportado na literatura (Barreiros et al., 2003; Martins et al., 2005; Chiou et al., 2006). A presença de co-específicos partidos em até três pedaços de tamanhos similares foi registrada nos estômagos dos indivíduos analisados. Em alguns casos, os tamanhos corporais dos co-específicos registrados como presas foram semelhantes ao do predador. A partição da presa em três pedaços pode ser um comportamento natural da espécie, e já foi registrado por Martins et al. (2005) que descreveram a alta voracidade de $T$. lepturus durante o canibalismo: antes de cortar as partes do corpo da presa através de mordidas para facilitar sua ingestão, o indivíduo utiliza suas maxilas para arrancar a cabeça do co-específico a ser consumido. Esse comportamento pode estar associado a morfologia corporal da espécie, principalmente do aparato bucal, facilitando a ingestão de presas de tamanhos variados, incluindo co-específicos de mesmo tamanho (Martins et al., 2005).

O comportamento de canibalismo é comum em peixes e alguns dos fatores que podem estar relacionados com a sua ocorrência se referem a: densidade populacional e diferenças de tamanho entre os co-específicos (Claessen e Roos, 2000; Dou et al., 2000; Atencio-Garcia e Zaniboni-Filho, 2006; Fessehaye et al., 2006), disponibilidade de alimento (Dou et al., 2000; Atencio-Garcia e Zaniboni-Filho, 2006) e características comportamentais (Fessehaye et al., 2006). Dou et al. (2000) descrevem que o canibalismo pode ser vantajoso para alguns indivíduos da espécie canibal, inclusive com benefícios nutricionais. Além disso, pode favorecer a redução da competição por espaço, alimento e parceiros sexuais.

Chiou et al. (2006) verificaram que o canibalismo em T. lepturus é acentuado quando há maior abundância de indivíduos na população ou escassez de presas. No sul 
do Brasil, Martins et al. (2005) relatam que o canibalismo é importante principalmente para os indivíduos adultos, podendo ocorrer devido a elevada abundância de presas co-específicas, comportamento gregário da espécie durante a alimentação e diminuição da produtividade biológica no ecossistema marinho daquela região nos meses de verão e outono. No norte do Rio de Janeiro, a escassez de presas parece não ser o fator que esteja conduzindo a espécie ao canibalismo (Di Beneditto et al, 1998; Di Beneditto, 2000; Di Beneditto et al., 2001) devido a alta produtividade biológica associada à desembocadura do Rio Paraíba do Sul. No entanto, os demais fatores não podem ser desconsiderados como explicativos para tal comportamento na região.

Apesar da predominância da piscivoria, os crustáceos peneídeos apresentaram relevância como item alimentar. A importância de crustáceos na dieta de $T$. lepturus já foi reportada para outras regiões (Martins et al., 2005; Chiou et al., 2006). A espécie P. muelleri, uma das presas preferenciais de $T$. lepturus na área de estudo, distribui-se desde áreas rasas até cerca de 600 metros de profundidade, em todo o Oceano Atlântico Ocidental (Costa et al., 2003), o que pode ser indicativo de que T. lepturus apresenta movimentação ao longo do gradiente batimétrico na região.

A espécie T. lepturus apresenta relevância ecológica na região estudada, tendo sido reportada como importante recurso alimentar para os cetáceos (Di Beneditto et al., 2001) e considerada como predador de topo de cadeia, consumindo espécies que são importantes como recursos pesqueiros na região, tais como: a pescadafaneca, Isopisthus parvipinnis; o goete, Cynoscion jamaiscensis; o pargo, Pargus pargus; a pescada-foguete, Macrodon ancylodon e os camarões barba-ruça, Artemesia longinaris e sete-barbas, Xiphopenaeus kroyeri. De acordo com os dados do presente estudo, T. lepturus pode ser considerada como uma das espécies chave na manutenção da estrutura e do funcionamento da cadeia trófica marinha do norte do Rio de Janeiro. No entanto, faz-se necessário a realização de estudos referentes ao fluxo de matéria e energia entre T. lepturus, suas presas, seus competidores e predadores, de modo a promover a elaboração de um modelo bioenergético para a espécie na região.

\section{Agradecimentos}

Agradecemos aos pescadores do porto de Atafona e a técnica de campo Silvana R. Gomes pelo auxílio nas coletas; a Dra ${ }^{\text {a }}$ Ilana R. Zalmon e ao Dr. Leandro R. Monteiro pelo auxílio; a FAPERJ (Proc. E-26/170.670/2004) pelos recursos financeiros; V.T. Bittar agradecece a Bolsa de Estudos conferida pela UENF e A.P. Di Beneditto agradece ao CNPq (Proc. 305160/2006-0) pela concessão de Bolsa de Produtividade em Pesquisa.

\section{Referências}

Aguiar, J. B. S. 2003. Influência da cadeia trófica marinha na ocorrência e abundância de peixes de importância comercial. Dissertação de Mestrado, Universidade Federal de Santa Catarina, Brasil, VI+98pp.

Atencio-Garcia, V.; Zaniboni-Filho, E. 2006. El canibalismo en la larvicultura de peces. Revista MVZ Córdoba, 11 (1): 9-19.

Barreiros, J. P.; Morato, T.; Santos, R. S.; de Borba, A. E. 2003. Seasonal changes in a sandy beach fish assemblage at Canto Grande, Santa Catarina, South Brazil. Journal of Coastal Research, 20 (3): $862-870$

Bastos, G. C. C. 1990. Morfologia de otólitos de algumas espécies de perciformes (Teleostei) da costa sudeste-sul do Brasil. Dissertação de Mestrado, Universidade de São Paulo/Instituto Oceanográfico, Brasil, 179pp.

Bassoi, M. 1997. Avaliação da dieta de toninhas, Pontoporia blainvillei (Gervais \& D'Orbigny, 1844), capturadas acidentalmente na pesca costeira de emalhe, no sul do Rio Grande do Sul. Monografia de Bacharelado, Fundação Universidade do Rio Grande, Brasil, 68pp.

Bassoi, M. 2005. Feeding ecology of franciscana dolphin, Pontoporia blainvillei (Cetacea: Pontoporiidae), and oceanographic processes on the Southern Brazilian coast. Tese de Doutorado, Graduate School of the National Oceanography Centre, USA, 207pp.

Boschi, E. E. 1963. Los camarones de la familia Penaeidae de la costa Atlantica de America del Sur. Ed. Instituto de Biologia Marina, Mar del Plata, Argentina, 39pp.

Cérvigon, F.; Cipriani, R.; Fisher, W.; Garibaldi, L.; Hendrickx, M.; Lemus, A. J.; Marquez, R.; Poutiers, J. M.; Robaina, G.; Rodríguez, B. 1993. Field guide to the commercial marine and brackishwater resources of the northern coast of South America. $1^{\text {st }}$ ed. FAO (Food and Agriculture Organization of the United Nations), Rome, Itália, 162pp.

Chiou, W. D.; Chen, C. Y.; Wang, C. M.; Chen, C. T. 2006. Food and feeding habits of ribbonfish Trichiurus lepturus in coastal waters of south-western Taiwan. Fisheries Science, 72: 373-381.

Claessen, D.; Roos, A. M. 2000. Bistability in a size-structured population model of cannibalistic fish-a continuation study. Theoretical Population Biology, 64: 49-65.

Clarke, M. R. 1986a. A handbook for the identification of cephalopod beaks. Claredon Press, Oxford, UK, 273pp. 
Clarke, M. R. 1986b. Cephalopods in the diet of odontocetes. In: Bryden, M. M. \& Harrison, R. (eds). Research of Dolphins. Claredon Press, Oxford, UK, p.281-321.

Costa, R. C.; Fransozo, A.; Melo, G. A. S.; Freire, F. A. M. 2003. Chave ilustrada para identificação dos camarões Dendrobranchiata do litoral norte do Estado de São Paulo, Brasil. Biota Neotropica, 3 (1) BN01503012003 Disponível em <http://www.biotaneotropica. org.br>. Acesso em 27 de janeiro de 2006.

Di Beneditto, A. P. M. 2000. Ecologia alimentar de Pontoporia blainvillei e Sotalia fluviatilis (CETACEA) na costa Norte do estado do Rio de Janeiro, Brasil. Tese de Doutorado, Universidade Estadual do Norte Fluminense, Brasil, 173pp.

Di Beneditto, A. P. M. 2003. Interactions between gillnet fisheries and small cetaceans in northern Rio de Janeiro, Brazil: 2001-2002. The Latin American Journal of Aquatic Mammals, 2 (2): 7986.

Di Beneditto, A. P. M.; Ramos, R.; Lima, N. R. W. 1998. Fishing activity on Northern Rio de Janeiro State (Brazil) and its relation with small cetaceans. Brazilian Archieves of Biology and Technology, 41 (3): 296-302.

Di Beneditto, A. P. M.; Ramos, R. M. A.; Lima, N. R. W. 2001. Os golfinhos: origem, classificação, captura acidental, hábito alimentar. Ed. Cinco Continentes, Porto Alegre, Brasil, 152pp.

Dou, S.; Seikai, T.; Tsukamoto, K. 2000. Cannibalism in Japanese flounder juveniles, Paralichthys olivaceus, reared under controlled conditions. Aquaculture, 182:149-159.

FAO (Food and Agricultural Organization) 2005. Disponível em: $<$ http://www.fao.org/figis/servlet/FiRefServlet?ds=species\&fid=24 68>. Acesso em 10 de maio de 2005.

Fessehaye, Y.; Kabir, A.; Bovenhuis, H.; Komen, H. 2006. Prediction of cannibalism in juvenile Oreochromis niloticus based on predator to prey weight ratio, and effects of age and stocking density. Aquaculture, 255: 314-322.

Figueiredo, L. L.; Menezes, N. A. 1978. Manual de peixes marinhos do sudeste do Brasil. II. Teleostei (1). $1^{\text {a }}$ ed. Museu de Zoologia de São Paulo, São Paulo, Brasil, 110pp.

Figueiredo, L. L.; Menezes, N. A. 1980. Manual de peixes marinhos do sudeste do Brasil. III. Teleostei (2). $1^{\text {a }}$ ed. Museu de Zoologia de São Paulo, São Paulo, Brasil, 90pp.

Figueiredo, L. L.; Menezes, N. A. 2000. Manual de peixes marinhos do sudeste do Brasil. VI. Teleostei (5). $1^{\text {a }}$ ed. Museu de Zoologia de São Paulo, São Paulo, Brasil, 90pp.

Fitch, J. E., Brownell, R. L., Jr. 1968. Fish otoliths in cetacean stomachs and that importance in interpreting feeding habits. Journal of the Fisheries Research Board of Canadian, 25 (12): 2561-2574.

GW-Basic 2.0, Programa. 1983. Copyright Microsoft 1983. AT\&T Personal Computer GW-Basic. CD-ROM. Copyright $<\mathrm{c}>1984$ by AT\&T, All right reserved.
Magro, M. 2006. Aspectos da pesca e dinâmica de populações do espada, Trichiurus lepturus (Trichiuridae, Teleostei), da costa Sudeste-Sul do Brasil. Tese de Doutorado, Instituto Oceanográfico, Universidade de São Paulo, Brasil, 174pp.

Magro, M.; Cergole, M. C.; Rossi-Wongtschowski, C. L. D. B. 2000. Avaliação do potencial sustentável de recursos vivos na zona econômica exclusiva - Revizee - Síntese de conhecimentos dos principais recursos pesqueiros costeiros potencialmente explotáveis na costa sudeste-sul do Brasil: peixes. MMA - Ministério do Meio Ambiente, dos Recursos Hídricos e da Amazônia Legal / CIRM - Comissão Interministerial para os Recursos do Mar, Brasília, Brasil, 145pp.

Magurran, A. E. 1988. Ecological diversity and its meansurement. Princeton University Press, Princeton, USA, 179pp.

Martins, A. S.; Haimovici, M. 1997. Distribution, abundance and biological interactions of the cutlassfish Trichiurus lepturus in the southern Brazil suptropical convergence ecosystem. Fisheries Research, 30: 217-227.

Martins, A. S.; Haimovici, M. 2000. Reproduction of the cutlassfish Trichiurus lepturus in the southern Brazil subtropical convergence ecosystem. Scientia Marina, 64 (1): 97-105.

Martins, A. S.; Haimovici, M.; Palacios, R. 2005. Diet and feeding of the cutlassfish Trichiurus lepturus in the Subtropical Convergence Ecosystem of southern Brazil. Journal of the Marine Biological Association of United Kingdom, 85: 1223-1229.

Menezes, N. A.; Figueiredo, L. L. 1980. Manual de peixes marinhos do sudeste do Brasil. IV. Teleostei (3). $1^{\text {a }}$ ed. Museu de Zoologia de São Paulo, São Paulo, Brasil, 96pp.

Menezes, N. A.; Figueiredo, L. L. 1985. Manual de peixes marinhos do sudeste do Brasil. V. Teleostei (4). $1^{\text {a }}$ ed. Museu de Zoologia de São Paulo, São Paulo, Brasil, 105pp.

Muehe, D.; Valentini, E. 1998. O litoral do Estado do Rio de Janeiro: uma caracterização físico-ambiental. Ed. FEMAR, Rio de Janeiro, Brasil, 99pp.

Pinkas, L.; Oliphant, M. S.; Iverson, I. L. K. 1971. Food habits of albacore, bluefin tuna zand bonito in Californian waters. Fish Bulletin, 152: 1-105.

Roper, C. F. E.; Sweeney, M. J.; Nauen, C. E. 1984. FAO Species Catalogue (Vol. 3) - Cephalopods of the World. Ed. FAO, Rome, Italy, 277pp.

Santos, M. C. de O.; Rosso, S.; Santos, R. A.; Lucato, S. H. B.; Bassoi, M. 2002. Insights on small cetacean feeding habits in southeastern Brazil. Aquatic Mammals, 28 (1): 38-45.

Wojciechowski, J. 1972. Observations on biology of cutlassfish Trichiurus lepturus L. (Trichiuridae) of Mauritania shelf. Acta Ichthyologica et Piscatoria, II (2): 67-75. 